\title{
Improvement in Oncological Care by Telemedical Support
}

At the break of the 21st century, medicine is headed for thorough changes. New molecular, biological and technological developments give rise to the hope that gentler, faster, more efficient and better diagnostic and therapeutic procedures become available for clinical application. Telemedicine is one of these new technologies. Telemedicine is neither a new diagnostic procedure nor a new oncological therapy. Nevertheless, from telemedicine we can expect profound improvements especially of oncological care.

Telemedicine offers passive or active functions in medical processes. An example for the passive application is the gathering of all medical findings in an electronic patient record. Without doubt, in- and outpatient care can be made more efficient in this way. Therefore, the introduction of this technique is regarded as a necessary prerequisite for maintaining medical services on a high level of quality even within a limited budget. In particular the tumor patient is characterized by a long-term patient career, which makes it difficult for the physician to be always kept sufficiently informed about all diagnostic and therapeutic results of and procedures for the individual patient. The possibility to have findings on the patient available at any given time can avoid burdens for the patient caused by doubled diagnostics, can improve the quality of medical care and can also keep the clinicians and practitioners involved collaboration at the same actual level of information - a cooperation which is not always easy. Also, the progressive specialization asks for telemedical techniques. Especially in oncology, the insight that medical expertise should be brought together in an interdisciplinary team and that therapeutic decisions should be made in common has long been accepted. By interactive video conferences, telemedicine will provide new forms of collaboration and medical consultations. Therefore it is essential to organically integrate the new techniques into the medical routine (e.g. an online second opinion).

Optimized tumor therapy is multidisciplinary and requires unhindered and rapid interdisciplinary interaction. Rather than the exchange of simple number-based data or verbal descriptions which more or less depict the patient status, interaction means an objective documentation of diagnostic findings. Highquality 3-D display of images based on video and computer technology enables such documentation. Via telecommunica- tion, this information can be transported practically in real time and to any place without loss of information. Only in this way, the non-repeatable particular situation in patient treatment can be depicted. This is necessary to gather the various bits of information in a time-relevant case and to be in a position to make the required multidisciplinary decisions.

As a spin-off, the documentation and accessibility of medical and administrative data offers the perspective for improvement of medical education and training in oncology.

Despite the availability of sufficient physical networks, until now the large-scale realization of telemedical applications in oncology failed because a coordinated introduction of infrastructure (standard user interface, etc.) was lacking. The compatible implementation of such an infrastructure is the main goal for the future. This should be considered within the context of a national telematic concept avoiding an isolated solution.

When telemedical techniques will play an active part in the medical processes and take over important issues in diagnosis and therapy of the tumor patients, also their role and potential will have to be evaluated critically. Thus, after initial enthusiasm, the discussion about expert systems that evaluate the digital patient information and give instructions to doctors on how to proceed, has become more restrained. The complexity in oncology can not be mastered only by computer support. However, telemedical techniques can contribute to increased diagnostic and therapeutic precision, thus reducing unnecessary and ineffective interventions. Besides the possibility of use for direct patient treatment, telemedicine will also indirectly influence medical services. Quality assessment in medicine will only become possible by widespread use of data processing based on telemedical techniques. Especially oncology plays a leading role with extensive meta-center studies and with the establishment of worldwide renowned and respected standards. The horror of a centralized and authoritarian governing system may arise here. However, telemedicine allows to establish a publicly accessible, distributed and standardized data base about illnesses and therapies. As can be seen, we have to critically deal with these new technologies from the very beginning to make full use of the chances offered and to steer the development into an ethically justifiable direction.

Peter M. Schlag, Berlin

KARGER $\quad \begin{aligned} & \text { (1) } 1999 \text { S. Karger GmbH, Freiburg } \\ & \text { Fax (0761) 4520714 } \\ & \text { www.karger.com } \\ & \text { Accessible online at: } \\ & \text { http://BioMedNet.com/karger }\end{aligned}$

